\title{
La diversidad en el aula: el desafío de interpretar la singularidad de los procesos de alfabetización inicial ${ }^{a}$
}

\author{
Diversity in the classroom: the challenge of interpreting the singularity \\ of initial literacy processes
}

\author{
Mónica O. Baez ORCID 0000-0003-2010-116X \\ María Eugenia D’Ottavio ORCID 0000-0001-9765-8340
}

Recibido: 4/10/2019 • Aprobado: 9/12/2019

Cómo citar: O. Baez, M., \& D’ Ottavio, M. E. (2020). La diversidad en el aula: el desafío de interpretar la singularidad de los procesos de alfabetización inicial. Ciencia y Educación, 3(3), 31-40. Doi: https://doi.org/10.22206/cyed.2019.v3i3.pp31-40

\section{Resumen}

La ley argentina Sobre Dificultades Especificas del Aprendizaje (Ley No 27.306, 2016) incluye entre sus principales puntos la dislexia como objeto de prevención y diagnóstico. Esto ha generado nuevos debates, así como el incremento de niños derivados a la consulta psicopedagógica desde las escuelas, con lo que se presenta el riesgo de una sobre-patologización de la población escolar, particularmente en el primer ciclo de la escolaridad primaria. Esto motivó este estudio cualitativo, basado en el marco interpretativo general de la epistemología y psicolingüística psicogenética, del que se da cuenta parcialmente en este artículo. Esta indagación se desarrolló en dos etapas: estudio de casos de niños derivados a atención psicopedagógica y exploración de las concepciones de una muestra de

\begin{abstract}
The Argentinian law on Specific Learning Difficulties (No 27.306, 2016) includes dyslexia as an object of prevention and diagnosis, among its main points. This has generated new debates as well as the proliferation of children referred from the schools to the psychopedagogical consultation, taking the risk of an over-pathologization of the school population, particularly in the first cycle of primary schooling. This motivated the present qualitative study, based on the general interpretative framework of psychogenetic epistemology and psycholinguistics, partially reported in this article. The research was developed in two stages: case studies of children referred to psychopedagogical care and exploration in a sample of teachers about their related conceptions. The results show that teacher training and
\end{abstract}

\footnotetext{
a El artículo se sustenta en datos y resultados obtenidos por una investigación realizada en la Universidad del Gran Rosario (Rosario, Argentina) entre 2015 y 2018 (Resoluciones Rectorales No 140/2014 y 005/2017). El equipo de investigación está conformado por las autoras (Coordinadoras del mismo) y los siguientes integrantes: Marcos Alionis, Florencia Cuneo, Marcela Gentile, Maira López, Soledad Domizi, Agustín Soria y Noelia Romero. Vale a ellos el agradecimiento y reconocimiento de las autoras por los aportes al equipo y a este artículo.

b Profesora e investigadora. Universidad Nacional de Rosario y Universidad del Gran Rosario, Argentina. Doctora en Ciencias en la Especialidad Investigaciones Educativas. Psicolingüista. Correo-e:monicaobaez1@gmail.com,monica.baez@unr.edu.ar

${ }^{\mathrm{c}}$ Profesora e investigadora. Universidad del Gran Rosario, Argentina. Licenciada en Psicopedagogía.

Correos-e: medottavio@gmail.com,psicopedagogía@ugr.edu.ar
} 
docentes. Los resultados manifiestan la importancia de la formación y de las intervenciones de los docentes para interpretar la normalidad de ciertas particularidades de los procesos de alfabetización.

Palabras clave: alfabetización; enseñanza de la lectura; enseñanza de la escritura; procesos de aprendizaje; dislexia.

\section{Introducción}

Las políticas educativas actuales y los cuerpos normativos vigentes en nuestro país, Argentina, así como en otros países latinoamericanos, fundamentan el imperativo de la integración e inclusión de todos los niños y jóvenes más allá de las diferencias físicas, culturales o de género. No obstante, muchos de ellos siguen siendo sujetos de mecanismos de exclusión escolar, sobre todo al comienzo y durante la escolaridad primaria; especialmente a partir de la evaluación escolar de sus prácticas de lectura y de escritura. Diferentes investigaciones (Unesco, 2010; Fundación Cimientos, 2011, entre otras) permiten determinar que los índices de repitencia y fracaso escolar se siguen incrementando, principalmente, en sectores de pobreza y de diversidad cultural.

La reciente Ley sobre Dificultades Especificas del Aprendizaje (Ley DEA), también conocida como Ley de Dislexia, promulgada en 2016 en Argentina, ha dado lugar al recrudecimiento de acciones discriminatorias que redundan en la patologización de muchos niños y jóvenes. Esto obedece a que bajo la innegable necesidad de prevenir e incluir, se postula no solo el carácter neurobiológico de esta dificultad (artículo 3), sino también la urgencia de detectarla de manera temprana, incluso antes del ingreso habitual al aprendizaje escolar de la lectura y de la escritura (artículos 5 y 8) habilitando con este fin a los docentes para su detección. Se insiste, además, en el trabajo con la lengua oral como prerrequisito para el ingreso a la escritura, a pesar de que numerosas investigaciones han demostrado que es el dominio de la escritura lo que permite la reformulación de la propia oralidad, y también se ha comprobado la incidencia del entorno sociocultural their intervention for interpreting the normality of certain particularities of literacy processes are both quite relevant.

Keywords: Literacy-Reading instruction; handwriting instruction; learning processes; dyslexia.

del alumno en la posibilidad de sus avances en ese conocimiento (Ferreiro, 2002, 2012; Blanche-Benveniste, 1982,1998, 2002).

En este contexto, ¿qué sucede con los niños de sectores vulnerables que tienen escasa ocasión de experimentar y reflexionar sobre las múltiples dimensiones significantes de la escritura en la escuela? ¿Qué sucede con los niños sordos cuya capacidad de aprender el español escrito como L2 ha sido demostrada por distintas investigaciones (Baez et al., 2009)? Prevención y salud, lectura y escritura, no son términos cuyas relaciones sean simples ni evidentes.

El interés por la prevención de la patologización de la infancia, problemática que se aborda parcialmente en este artículo, es de larga data en el campo de la Psicopedagogía y de las disciplinas vinculadas al campo del aprendizaje. En cuanto a la denominación "dislexia”, según Fusca (2016), funciona como un "paraguas" que se usa para encubrir una gran variedad de problemas. Una gama cada vez más amplia de dificultades cognitivas, problemas de atención, dificultades de análisis y síntesis, dificultades en la organización y expresión de ideas. De modo que "dislexia", es un término confuso ya que lo que para un profesional es dislexia para otro no lo es.

Al respecto, en una conferencia de 1975, la Dra. Emilia Ferreiro, investigadora argentina, advertía sobre los trastornos de aprendizaje producidos por la escuela. En ese trabajo, de una vigencia notable en nuestros días, Ferreiro exponía dos tesis: la primera se refiere a que "nuestra manera de evaluar un trastorno de aprendizaje depende de la concepción que sustentamos acerca del proceso de aprendizaje", la segunda: "hay trastornos de aprendizaje producidos por la escuela”. El presente trabajo se orienta a la elucidación 
de la actualidad de dichas tesis, focalizándonos en la necesidad de atender a los desarrollos en investigación psicolingüística de base psicogenética (Ferreiro y Teberosky, 1979; Ferreiro, 1987, 1990, 1999 entre otros, y en adelante) y didáctica socio-constructivista (Lerner, 1987, 2001; Kaufman y Perelman, 2000; Nemirovsky, 2004, por ejemplo) de los últimos años, que permiten reinterpretar las perspectivas tradicionales acerca de la alfabetización inicial.

\section{Marco teórico de referencia}

La alfabetización es un problema escolar que trasciende a la escuela como institución. Constituye un tema central que es posible redimensionar desde distintas perspectivas: por un lado, en términos de las prácticas y saberes que la cultura letrada de hoy exige; por el otro, la resignificación de temas y problemas sobre la temática, a partir de los aportes de distintas disciplinas. En este sentido, nos parece necesario tener en cuenta las investigaciones psicolingüísticas de base psicogenética (Ferreiro y Teberosky, 1979; Ferreiro, 1975, 1999, entre otros) desarrolladas a lo largo de más de treinta años respecto de cómo aprenden los niños a leer y a escribir, los aportes de la historia de las culturas escritas (Chartier, 1994, 2000, 2019; Cavallo y Chartier, 2001; Petrucci, 2003; Olson, 1998, por citar), de la sociología de la lectura (Lahire, 2004), de la lingüística (Blanche-Benveniste, 2002); disciplinas que, entre otras contribuciones, alentaron el desarrollo de una didáctica de la lectura y de la escritura fundada en los procesos conceptuales de un sujeto en desarrollo.

A partir de la historia de la escritura, sabemos que su aparición, así como también su desarrollo como una modalidad de lenguaje particular, estuvo ligada a usos y prácticas sociales con fines determinados, al que no fueron ajenos los cambios tecnológicos. El dominio de la escritura obliga a comprender un uso de la lengua caracterizado por convenciones formales, lingüísticas, discursivas y gráficas constituidas a través de la historia y de las prácticas culturales, que no se corresponden con el lenguaje oral cotidiano. En términos de Blanche-Benveniste (1982, pp. 247-270) se trata de un "lenguaje dominguero", que escapa, por sus funciones e historia, a la noción de "código escrito" sostenida tradicionalmente.

Los sistemas de escritura actuales son objetos sociales y culturales que derivan de alguno de los pocos sistemas originales que se han producido a lo largo de la historia — como sabemos el sistema de escritura alfabético proviene del sumerio - transformándose y adaptándose a las características de diferentes lenguas que incidieron en las reglas y principios adoptados por cada uno de ellos. El reconocimiento de la historicidad de este objeto particular fue fundamental en el desarrollo de las investigaciones psicolingüísticas que sirven de referencia a nuestro trabajo. En palabras de Ferreiro (1996, p. 184):

Para tratar de comprender las razones de las respuestas insólitas de los niños recurrí a la historia de la escritura en la humanidad. El recurso a la historia también es una herencia piagetiana. Lo aprendí en el Centro de Epistemología Genética, y desde entonces no he cesado de apreciar la profundidad de esa enseñanza.

Las hipótesis infantiles descubiertas, descritas y corroboradas desde esas indagaciones en numerosas poblaciones de niños que aprenden sistemas de escritura alfabéticos (español, portugués, francés, italiano, por ejemplo) constituyen respuestas lógicas a los desafíos que el sistema de escritura alfabético les plantea, a medida que sus posibilidades de coordinación y diferenciación, es decir, de operar cognitivamente con el objeto, se complejizan a lo largo de su desarrollo. Ninguna de las hipótesis que el niño se formula (distinción y relación entre letras y números, hipótesis del nombre, de cantidad, de variedad, silábica $y$, finalmente, alfabética) es azarosa. El empleo del término "hipótesis" es significativo, pues manifiesta el reconocimiento en el niño de ideas, de nociones, de conceptualizaciones propias, es decir, la convicción de que se trata de un sujeto activo cuyos aprendizajes escolares no comienzan de cero.

De modo que, desde esta perspectiva, las producciones y argumentos de los niños no se interpretan en función de rendimiento o eficacia, como suelen plantear algunas investigaciones actuales sobre la lectura, ni en términos de correcto o incorrecto; posturas estas que implican la atención al producto. 
A partir de estos trabajos sabemos el valor y el significado de producciones y argumentos de los alumnos, que desde el punto de vista de los adultos alfabetizados pueden constituir errores, pero que desde el punto de vista del sujeto que aprende son necesarios para la reinvención conceptual de este objeto social, histórico y lingüístico complejo, la escritura.

Dichos avances han permitido resignificar los parámetros desde los que se juzga la normalidad o patología que ponen de manifiesto sujetos en situación de aprendizaje del lenguaje escrito y, a su vez, con ello, es posible interrogar los criterios y prácticas que contribuyen a promover la inclusión educativa. Dado que la alfabetización es inherente a los procesos de educación de cualquier nivel y modalidad, el análisis de esta permite recontextualizar el papel de las instituciones educativas, del docente y el de los profesionales vinculados a ese quehacer.

Estas consideraciones nos permiten reubicar en una nueva perspectiva de interpretación al aprendizaje de la lectura y de la escritura. Particularmente, el de aspectos inherentes a la circulación de lo escrito que se constituyen en datos a la hora de definir la marcha escolar de un niño. Por lo tanto, y desde la perspectiva epistemológica y psicolingüística asumida, se plantea como un desafío la indagación de los aciertos (entendidos como modos de "saber") y no los errores o carencias que se ponen de manifiesto en los argumentos y en las producciones escritas infantiles. El uso no convencional de aspectos gráficos, ortográficos y textuales, que reflejan los modos por los cuales los niños comprenden y organizan un texto, es considerado con mucha frecuencia como indicador sintomático de una patología específica —dislexia, con frecuencia - o bien como índice de una no adecuación a los parámetros escolares vigentes. Resulta necesario entonces rescatar el significado que poseen los haceres y quehaceres de los nińos con el lenguaje escrito. Proponemos entonces, volver la mirada al niño en tanto sujeto de conocimiento y, en términos piagetianos, interlocutor intelectual válido. También a los docentes, en tanto sujetos de aprendizaje y de conocimiento, quienes no siempre cuentan con las oportunidades adecuadas para resignificar los parámetros desde los que se ha formado, y aún se forman, o las novedades que surgen en su campo profesional.
Si por aprendizaje entendemos método de enseñanza, si el método se basa en una sucesión acumulativa de retazos de saber que de manera transparente son transmitidos y se espera que de igual manera sean reproducidos, sin interpretar la mediación psicológica del sujeto que aprende y sin tener en cuenta la incidencia de las condiciones culturales y didácticas que atraviesan tanto a la enseńanza como al aprendizaje, los errores de los alumnos solo pueden categorizarse como fallas —clínicas o sociales- de los alumnos y/o de sus familias. Sin embargo, ya Piaget nos mostró la originalidad y pertinencia de la actividad lógica y constructiva que desarrollan los niños para reconstruir el mundo, así como la distancia compleja que media entre la enseńanza y el aprendizaje.

Los modelos didácticos fundados en el etiquetamiento surgen como una necesidad de ordenar y controlar lo no interpretable, en búsqueda de un ideal de homogeneización siempre inalcanzable. La escuela corre entonces el riesgo de verse impulsada a abandonar su lugar esencial en la formación de ciudadanos críticos para apropiarse de etiquetas clínicas detrás de las cuales el alumno como "interlocutor intelectual válido” (Ferreiro, 1999) queda elidido.

\section{Objetivos del estudio}

A partir de lo antedicho asumimos la indagación de un recorte de la problemática señalada que se sintetiza en los siguientes objetivos:

- Profundizar la indagación de los procesos que desarrollan los niños en vías de alfabetización derivados a la consulta psicopedagógica, para problematizar los pre-diagnósticos que argumentan su derivación bajo la categoría de dislexia.

- Indagar las concepciones de lectura y de escritura que sustentan las decisiones de los docentes al solicitar la atención psicopedagógica en dichos casos.

\section{Metodología}

La metodología adoptada, basada en un enfoque cualitativo, se funda en el método de exploración crítica (Piaget, 1970). La misma provee una mirada 
particular a partir de la cual el conocimiento no es un estado invariante, sino un proceso cuyo devenir es necesario analizar con la mayor exactitud posible (Piaget, 1976). La reconstrucción de ese devenir, que permite determinar cuáles son las relaciones entre un sujeto y un objeto de conocimiento particular, requiere de diferentes estrategias metodológicas que permitan explorar, desde la perspectiva de los niños, los sistemas de creencias. Debido a esto, se optó por un diseño de investigación flexible y permeable a lo que acontece en el campo, brindándonos la posibilidad de construir categorías no previstas.

Este estudio se diseñó teniendo en cuenta dos tipos de población: niños derivados a la consulta psicopedagógica gratuita, que se ofrece en la Unidad Docente Asistencial (UDA) dependiente de la Licenciatura en Psicopedagogía de la Universidad del Gran Rosario (Rosario, Argentina), y docentes en actividad a cargo de $1^{\circ}, 2^{\circ}$ o $3^{\circ}$ grado de la escolaridad primaria (Primer Ciclo). A la vez, se desarrolló en dos etapas. La primera etapa se focalizó en un estudio de casos que posibilitó el seguimiento de diez nińos que llegaron a UDA con pre-diagnósticos escolares de patologías vinculadas al aprendizaje de la lectura y de la escritura. La segunda, en el desarrollo de entrevistas en profundidad y de una situación experimental consistente en el análisis e interpretación de escrituras de niños en proceso de alfabetización con treinta docentes seleccionados a tal efecto. Respecto de la primera etapa, reiteramos que los nińos con los que trabajamos llegaron al UDA por solicitud de sus docentes en todos los casos. Se trataba de alumnos que cursaban la escolaridad primaria en escuelas públicas o de gestión privada y provenían de localidades vecinas a Rosario o de la periferia de esta ciudad; pertenecían a entornos familiares de bajos recursos. Las sesiones, con una secuencia de entrevistas quincenales o mensuales — según las asistencias de los niños-, se focalizaron en situaciones de escritura, revisión de escrituras, reescrituras, edición de textos en computadora y de lectura (búsqueda de información, identificación de aspectos paratextuales, etc.) con portadores de textos reales. De modo que, se propusieron trabajos de lectura y de escritura que permitían interrogar el discurso patologizante que acompañaba a cada niño.
En la segunda etapa se trabajó con docentes de nivel primario de la educación básica, que estaban dictando clases en el primer ciclo en instituciones de la ciudad de Rosario. La fundamentación de la elección de la muestra se encuentra basada en el posicionamiento de que en los primeros grados los niños están en un proceso de construcción de la lengua, tanto escrita como hablada, y se estima que no debería ser posible realizar una derivación a tratamiento específico en estos momentos fundantes. Los docentes entrevistados aludieron a sus concepciones acerca de la lectura, la escritura, factores que favorecen u obstaculizan dichos aprendizajes, modalidad de enseñanza, las que fueron corroboradas a través del análisis crítico e interpretación de las escrituras infantiles propuestas.

Se optó por las dos situaciones de indagación citadas - entrevistas en profundidad y situación experimental-, a fin de acceder a las teorías implícitas de los mismos en relación con la enseńanza de la lectura y la escritura, y de esta manera acercarnos y explorar los modos de intervención que consideran posibles frente a alumnos que presentan escrituras no convencionales.

\section{Resultados}

Por razones de espacio, respecto de la primera etapa - estudio de casos - aquí nos centraremos en uno de los niños indagados, ya que ilustra adecuadamente lo sucedido con otros. Lo llamaremos Simón, quien, en el momento de realización del estudio, contaba con 8,2 ańos y cursaba $3^{\circ}$ grado de nivel primario en una escuela de gestión privada de una ciudad aledaña. Este niño, como los otros niños indagados, cuenta con una familia comprometida con su desarrollo, preocupada por él, perteneciente a sectores en vulnerabilidad social, entornos con escasas posibilidades de interacción con material escrito. Simón fue derivado por recomendación de la maestra, quien sostenía que: "tiene dificultades de aprendizaje, dislexia en particular, porque omite letras al escribir, y problemas neurológicos, se distrae y no puede leer letra de imprenta minúscula, no puedo hacer nada con él" Sugería la asistencia de profesionales: psicopedagogo, 
fonoaudiólogo y neurólogo. Estos dichos fueron repetidos por la mamá del niño en la entrevista que mantuvimos con ella, y por el propio niño al inicio de la primera sesión, quien sostuvo: "a mí me dan mucha tarea porque no sé leer en imprenta minúscula”. Ya se ha instalado en el discurso que lo designa como "otro" desde el lugar del que no puede o no debe, el "no puede" de la maestra es asumido por el niño en primera persona.

Los cuadernos de Simón revelan propuestas didácticas basadas en el uso de fotocopias para situaciones de lectura y de escritura y de escasísimas escrituras propias, básicamente ritualizadas (fecha, nombre, etc.). Los fragmentos del estudio que aquí referiremos son recortes de la secuencia más amplia de las tareas llevadas a cabo con este niño.

La primera tarea que propusimos a Simón fue la escritura de una lista de compras para el supermercado, con el propósito de indagar acerca del nivel de conceptualización de escritura del nińo, para lo cual, luego de pedirle que escriba su nombre, contextualizando la situación se le dictaron una serie de palabras seleccionadas según su estructura silábica $\mathrm{CV} / \mathrm{CCV} /$ CVC/CVVC y la acentuación de las mismas. Este criterio se fundamentó en la necesidad de interpretar qué tipo de hipótesis sostiene el niño respecto de la representación alfabética, ya que las que llamamos sílabas compuestas problematizan ese principio.

Gráfico 1. Escritura de lista de compras (Simón, 8:2 años)

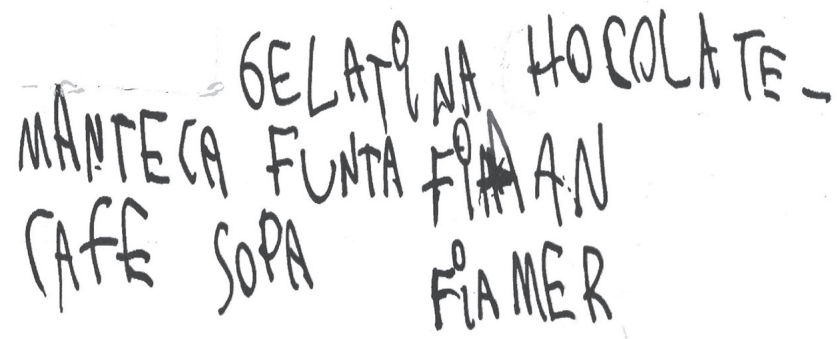

Respecto de la escritura de estas palabras, observamos regularidades desviantes ya puestas de manifiesto en otros estudios, en tanto indicadoras de los procesos de organización interna del niño (Brown, 1973 en Ferreiro 2013, p. 134), es decir, conceptualizaciones que Simón construye intentando comprender cómo funciona nuestro sistema de escritura en el caso específico en que una palabra incluye una sílaba constituida por CCV, poniendo en tensión el análisis silábico con el análisis fonológico, ya que se altera la estructura CV. Esto se ve en ciertas omisiones (en "chocolate"), sustituciones y cambio de lugar de las consonantes (en "fruta" y "fiambre"). El problema no se centraría en la identificación de las consonantes, dado que lee cada palabra correctamente, sino en la necesidad de sostener el principio alfabético propio de nuestra escritura. En el momento de revisión de sus propias escrituras, se autocorrige y agrega de manera pertinente las consonantes necesarias según la secuencia esperada. En este momento Simón parece estar preocupado por este tipo de estructuras silábicas concentrándose en un análisis intrasilábico.

Gráfico 2. Revisión de lista de compras (Simón, 8,2 años)

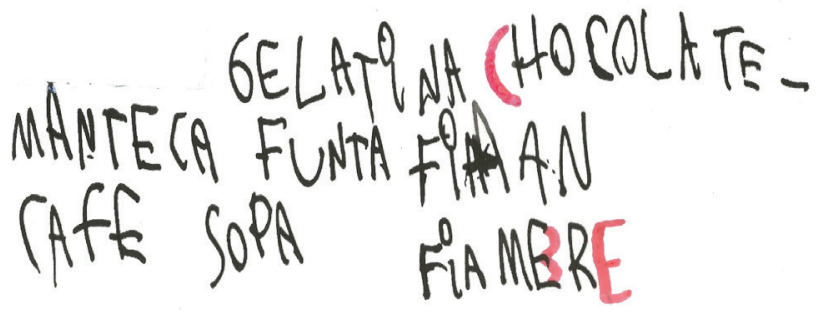

Quince días después su escritura muestra haber superado la problemática que le generaba el dígrafo "ch" y su preocupación parece haberse desplazado del análisis intrasilábico a uno la atención a los límites de la sílaba, es decir, a las interacciones intersilábicas. Así, cuando le propusimos la escritura de las instrucciones para hacer un burbujero - en las que consideramos dificultades semejantes a las propuestas en la primera situación - sistemáticamente Simón no escribe una de las consonantes que coinciden en el límite entre una sílaba y otra al interior de las palabras en cuestión. 
Gráfico 3. Burbujero (Simón, 8,2 años)

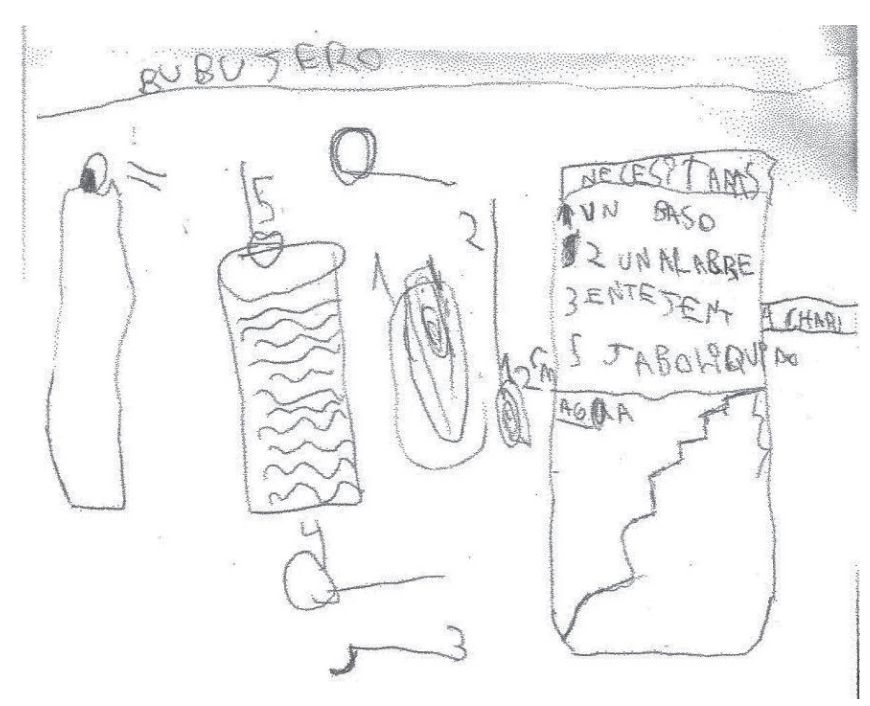

Las omisiones de las consonantes -observables desde la mirada de un adulto alfabetizado-, muestran regularidad: bubujero (por burbujero), alabre (por alambre), chapú (por champú), jaboliquido (por jabón líquido, en este caso, — más allá de la hipo-segmentación, habitual en los inicios de la alfabetización-, coinciden otra vez dos consonantes en final de una sílaba y comienzo de otra, pero en este caso en situación interpalabra), detejete (por detergente). En este momento del proceso de alfabetización de Simón, esas realizaciones y la regularidad de las mismas obedecerían a una lógica propia de un niño que está re-inventando activamente las unidades (sílaba/palabra) a la par de los principios que el sistema impone. Reconstruir el principio alfabético —esa correspondencia ideal grafema/fonema- es un logro muy fuerte que interactúa con otros conceptos involucrados en la escritura, como el de la reconstrucción de las unidades de lo escrito y, con ello, los tipos de estructura silábica que presenta nuestra escritura.

Por otra parte, este mismo niño, en las situaciones de lectura que le propusimos desde las primeras sesiones - búsqueda de cuentos a partir de los títulos escritos en imprenta mayúscula y minúscula en distintas etiquetas, por ejemplo-, mostró claramente y para su propia sorpresa que leía listas de palabras, índices, títulos de cuentos y cuentos tanto en imprenta mayúscula como en minúscula. Vimos también que la posibilidad de actuar como revisor de su propia escritura le permitió corregirla y asumir su responsabilidad sobre la misma (Lerner, 2001). También la disponibilidad de materiales escritos diversos y reales en contextos significantes, promovió procesos de reflexión metalingüística acerca de aspectos tipográficos y dispositivos textuales ausentes en las fotocopias y en las escrituras fragmentadas que muestra su experiencia escolar. Los diferentes profesionales que integran interdisciplinariamente el equipo de investigación corroboraron la ausencia de problemas neurológicos, fonoaudiológicos (solo manifestaba respiración bucal), psicolingüísticos y psicopedagógicos ya que estaba en pleno proceso de alfabetización. Luego de tres meses de iniciado nuestro estudio, Simón leía y escribía de manera convencional. Este avance fue constatado en el resto de los niños indagados.

En cuanto a la indagación desarrollada con los docentes, nos referiremos brevemente a una parte de los datos obtenidos a través de entrevistas realizadas en profundidad con estos, y de la propuesta de interpretar escrituras infantiles no convencionales pertenecientes a niños sin patología; todo esto nos ha permitido interpretar las dificultades que plantea la persistencia de paradigmas que tienden a la homogeneización de la enseñanza y de los aprendizajes. Pudimos determinar en la muestra indagada que, en cuanto a los fines de la enseńanza de la lectura y de la escritura, para el $42 \%$ de los entrevistados la importancia radica en la idea de una alfabetización funcional (inserción social, obtener trabajo, etc.), para un $48 \%$ los propósitos de este aprendizaje aparecen vinculados a fines escolares, y solo para un $20 \%$ de la muestra sostiene diversidad de propósitos: resolución de problemas, búsqueda de información, disfrutar de textos literarios, etc. En cuanto a la conceptualización de las actividades de leer y de escribir en sí mismas, un 46 \% revela una formación coherente con una perspectiva conductista, basada en criterios codificación-decodificación y propuestas descontextualizadas, un $34 \%$ manifiesta nociones contradictorias entre sí y un $20 \%$ asume una perspectiva basada en las posibilidades de significación e interpretación que ofrece el lenguaje escrito y la importancia de recrear en el aula las prácticas que caracterizan a un mundo letrado. 
Es interesante destacar que el $82 \%$ de los docentes entrevistados da cuenta de conocer los aportes de las investigaciones psicolingüísticas de base psicogenética y de la didáctica socio-constructivista, desde las que se ofrecen pistas para interpretar y promover la actividad cognitiva de los sujetos en proceso de aprendizaje. Sin embargo, al momento de responder cómo aprenden sus alumnos, solo el $22 \%$ de los docentes manifiestan interpretar la actividad constructiva y la complejidad de los procesos cognitivos que llevan a cabo sus alumnos, además de destacar la importancia de enseñar a través de situaciones significativas que les permitan a los niños vincular los contenidos pedagógicos con experiencias vividas. Ańadiendo que enseñar las letras por separado de manera descontextualizada no permite que los niños comprendan los propósitos reales de comunicación que presentan los textos. Por ello, en el aula utilizan diferentes tipos de textos a la hora de enseñar. Finalmente, enfatizaron el valor del trabajo en grupo ya que les permite a los niños intercambiar ideas y opiniones acerca de los textos brindados por el docente y ayudarse o corregirse mutuamente e interactuar con sus pares desde niveles diferentes de conceptualización. En cambio, el $48 \%$ se limita a aclarar que los nińos aprenden a leer y a escribir por la maduración y la motivación con la que cuentan al momento del ingreso a la escolaridad primaria. Pareciera, según los discursos de estos docentes, que para que el niño aprenda deben conjugarse una estructura biológica óptima, el permanente acompañamiento familiar e intervenciones docentes basadas en estímulo, control y evaluación. En este enfoque se escinde del proceso al propio sujeto del aprendizaje, a los procesos psicológicos y cognitivos que este construye en pos de conocer e interpretar al mundo que lo circunda y la incidencia del entorno sociocultural en los puntos de partida.

Respecto de lo que aparecen como dificultades de aprendizaje, solo el $22 \%$ de los entrevistados plantea la posibilidad de que esas dificultades se deban a la metodología de enseñanza reproducida y sostenida por la institución escolar. Finalmente, al encontrarse con la situación experimental (producciones de niños en diversos momentos del aprendizaje del lenguaje escrito), el $50 \%$ de los docentes señaló que las escrituras eran aceptables, aunque no convencionales, en un momento del desarrollo determinado, pero dejan de lado la posibilidad de analizar e interpretar el significado de esos aspectos no convencionales desde la perspectiva de lo que el niño muestra saber, también de mencionar las situaciones didácticas que le permitirían avanzar hacia una escritura cada vez más convencional. Ese porcentaje, como los antes citados, habilitaría la sospecha de que sigue vigente la segunda tesis formulada por Ferreiro (1975): "hay trastornos de aprendizaje producidos por la escuela".

\section{Conclusiones}

En los ejemplos que nos ofrecen los decires de los docentes entrevistados y los de las maestras que solicitaron la atención psicopedagógica de los niños estudiados — como el citado de la maestra de Simónobservamos que ciertas teorías implícitas subyacentes a esos discursos, en muchos casos articulados con ciertas creencias extraescolares, obstaculizan la posibilidad de interpretar los procesos de alfabetización en términos de construcción de saberes, en vez de una sucesión de fallas o carencias. La maestra de Simón no pudo "ver", es decir, interpretar, las manifestaciones de esos procesos como aprendizaje, y a los errores en las escrituras del niño como un modo de saber, no de manera intencional, sino porque los discursos que han constituido su rol están anclados a un modelo didáctico que los ubica necesariamente en la función de etiquetar: hoy disléxicos, en otro tiempo inmaduros, e incluso, hubo un tiempo de aulas con un número preciso de pre-silábicos cuyo pasaje al grado o nivel siguiente era puesto en duda. Esto se pone de manifiesto en los decires de la maestra de Simón, replicándose en varios de los docentes entrevistados, al expresar preocupación acerca de las producciones escritas del nińo, lo que anima a la continuidad y consolidación de esta lógica de etiquetamiento.

Es posible y necesario promover otro tipo de formación profesional, que posibilite el "error" en los propios procesos de aprendizaje de los docentes, que les permita tematizar las contradicciones propias de toda construcción de saberes nuevos, a pesar de que la reformulación de esos modos de significación supone un serio y difícil camino, no libre de resistencias y contradicciones, como todo aprendizaje. 
Proceso posible y necesario a condición de que, como consideramos para los nińos, se creen y promuevan el conjunto de medidas indispensables para que los docentes cuenten con las condiciones propicias para llevarlo a cabo. Una escuela inclusiva requiere de una comunidad educativa que disponga de herramientas conceptuales que les permita dar sentido educativo y didáctico a la diversidad, siempre presente en las aulas. ¿Por qué no asegurar la constitución de un rol profesional docente quien, como parte de un equipo interdisciplinario, pueda asumir la especificidad de su quehacer? ¿Por qué no capacitar a los profesionales de la salud en lo que hoy sabemos acerca de los procesos de aprendizaje? ¿Por qué no luchar por una escuela que no requiera del adjetivo de inclusiva, es decir, un espacio donde se asuma la diversidad y la singularidad inherentes a lo humano como evidencia y como ventaja pedagógica?

Cuando la pregunta se desplaza de "cómo hacer para que mis alumnos pasen de un estado de menor conocimiento a uno de mayor conocimiento" hacia "cómo catalogo al que no va con lo esperado", cuando la demanda de ayuda se focaliza en contar con herramientas para diagnosticar antes que para enseñar, la interpretación - y celebración — de la diversidad lejos de promoverse se vuelve utopía y la alfabetización se vacía de significación.

\section{Referencias}

Baez, M. et al., (2009). Diálogos con sordos. Aportes para reinterpretar la alfabetización de Sordos. Rosario, Argentina: Laborde Ed.

Blanche-Benveniste, C. (1982). "L'image de la norme linguistique propre aux textes écrits. Les différences entre cette norme et les normes de la langue quotidienne." En Noves perspectives sobre la representació escrita en el nen. Barcelona, España: IME/ICE.

Blanche-Benveniste, C. (1998). Estudios lingüisticos sobre la relación entre oralidad y escritura. Barcelona, España: Gedisa.

Blanche-Benveniste, C. (2002). "La escritura, irreductible a un 'código"'. En: E. Ferreiro (comp.). Relaciones de (in) dependencia entre oralidad y escritura (pp. 15-30). Barcelona, España: Gedisa.
Cavallo, G. y Chartier, R. (2001). Historia de la lectura en el mundo occidental. Madrid, España: Taurus-Minor.

Chartier, R. (1994). El orden de los libros. Lectores, autores, bibliotecas en Europa entre los siglos XIV y XVIII. Barcelona, España: Colección LEA, Gedisa.

Ferreiro, E. y Teberosky A. (1979/1999). Los sistemas de escritura en el niño. México D.F, México: Siglo XxI.

Ferreiro, E. y Teberosky, A. (1975). Problemas de Psicologia Educacional. Buenos Aires, Argentina: IPSE.

Ferreiro, E. y Teberosky, A. (1999). Cultura escrita y educación. Conversaciones con Emilia Ferreiro. México D.F, México: Fondo de Cultura Económica.

Ferreiro, E. y Teberosky, A. (2002). Relaciones de (in) dependencia entre oralidad y escritura. Barcelona, España: Gedisa.

Ferreiro, E. y Teberosky, A. (1987). "El aprendizaje en el niño y los aprendizajes escolares". En N. Elichiry (comp.) El niño y la escuela. Reflexiones sobre lo obvio. Buenos Aires: Nueva Visión.

Ferreiro, E. y Teberosky, A. (1990). Proceso de alfabetización. Alfabetización en proceso. Buenos Aires, Argentina: CEAL.

Ferreiro, E. y Teberosky A. (1996). "Aplicar, replicar, recrear: acerca de las dificultades inherentes a la incorporación de nuevos objetos al cuerpo teórico de la teoría de Piaget". Substratum, 3(8-9), 181.

Ferreiro, E. (1997) “La noción de palabra y su relación con la escritura” en R. Barriga y P. Butragueño (eds.), Varia lingüística y literaria. 50 años del CELL. Lingüística, I, (pp. 343-361). Ciudad de México, México: El Colegio de México.

Ferreiro, E. y Teberosky, A. (1999). Vigencia de Jean Piaget. México D.F., México: Siglo xxi.

Ferreiro, E. y Teberosky, A. (2000). Pasado y presente de los verbos leer y escribir. Buenos Aires, Argentina: Fondo de Cultura Económica.

Ferreiro, E. y Teberosky, A. (comp.). (2002). Relaciones de (in) dependencia entre oralidad y escritura. Barcelona, España: Gedisa. 
Ferreiro, E. et al., (2000). Sistemas de escritura, constructivismo y educación, a veinte años de la publicación "Los sistemas de escritura en el desarrollo del niño". Rosario, Argentina: Homo Sapiens ediciones.

Fundación Cimientos (2011) La educación argentina en números. Actualización de datos estadisticos sobre la escolarización en Argentina a abril de 2011. Documento No 6. Buenos Aires, Argentina. Disponible en: http://www.cimientos.org/ archivos/educacion_6.pdf

Fusca, C. (2016). ¿Qué es eso llamado Dislexia? Buenos Aires, Argentina: Revista Noveduc.

Fusca, C. (2004). Los niños piensan sobre la escritura. cd-rom. México D.F., México: Siglo xxi.

Fusca, C. (2013). El ingreso a la escritura y a las culturas de lo escrito. México D.F., México: Siglo Xxi.

Kaufman, A. M. y Perelman, F. (2000) "Resúmenes escritos: reconstrucción de un proceso investigativo" en M. Elichiry, (comp.) Aprendizaje de niños y maestros. Hacia la construcción del sujeto educativo. Buenos Aires, Argentina: Manantial.

Lahire, B. (2004). El hombre plural. Barcelona, España: Bellaterra.

Lerner, D. (2001). Leer y escribir en la escuela: lo real, lo posible y lo necesario. México D.F., México: Fondo de Cultura Económica.

Lerner, D. (1987). Encuentro Latinoamericano sobre experiencias alternativas en Alfabetización de niños. México, México D.F: Organización de Estados Americanos.
Nemirovsky, M. (2004). «Escribimos en el aula, según en qué aula». Aula de Infantil, (18), 5-9.

Olson, D. (1998). El mundo sobre el papel. El impacto de la escritura y la lectura en la estructura del conocimiento. Barcelona, España: Gedisa.

Petrucci, A. (2003). La ciencia de la escritura. Buenos Aires, Argentina: Fondo de Cultura Económica.

Piaget, J. (1993). La representación del mundo en el niño. Madrid, Espańa: Ediciones Morata.

Piaget, J. (1976). La toma de conciencia. Madrid, España: Morata.

Piaget, J. (1970/1981). Piaget's Theory, en Carmichael's Manual of Child Psychology. New York, Estados Unidos: Wiley.

Senado y Cámara de Diputados de la Nación Argentina. (19 de octubre de 2016). [Ley No 27.306], Dificultades Específicas del Aprendizaje. Boletin Oficial de la República Argentina. Buenos Aires, Argentina.

UNESCO. (2010). EPT Informe de seguimiento 2010. Llegar a los marginados. París, Francia. Disponible en: www.unesco.org/es/efareport/ reports/2010-marginalization/ 